\title{
revmaterialeplastice.ro
}

Vol. 57 / Number 3 / Year 2020
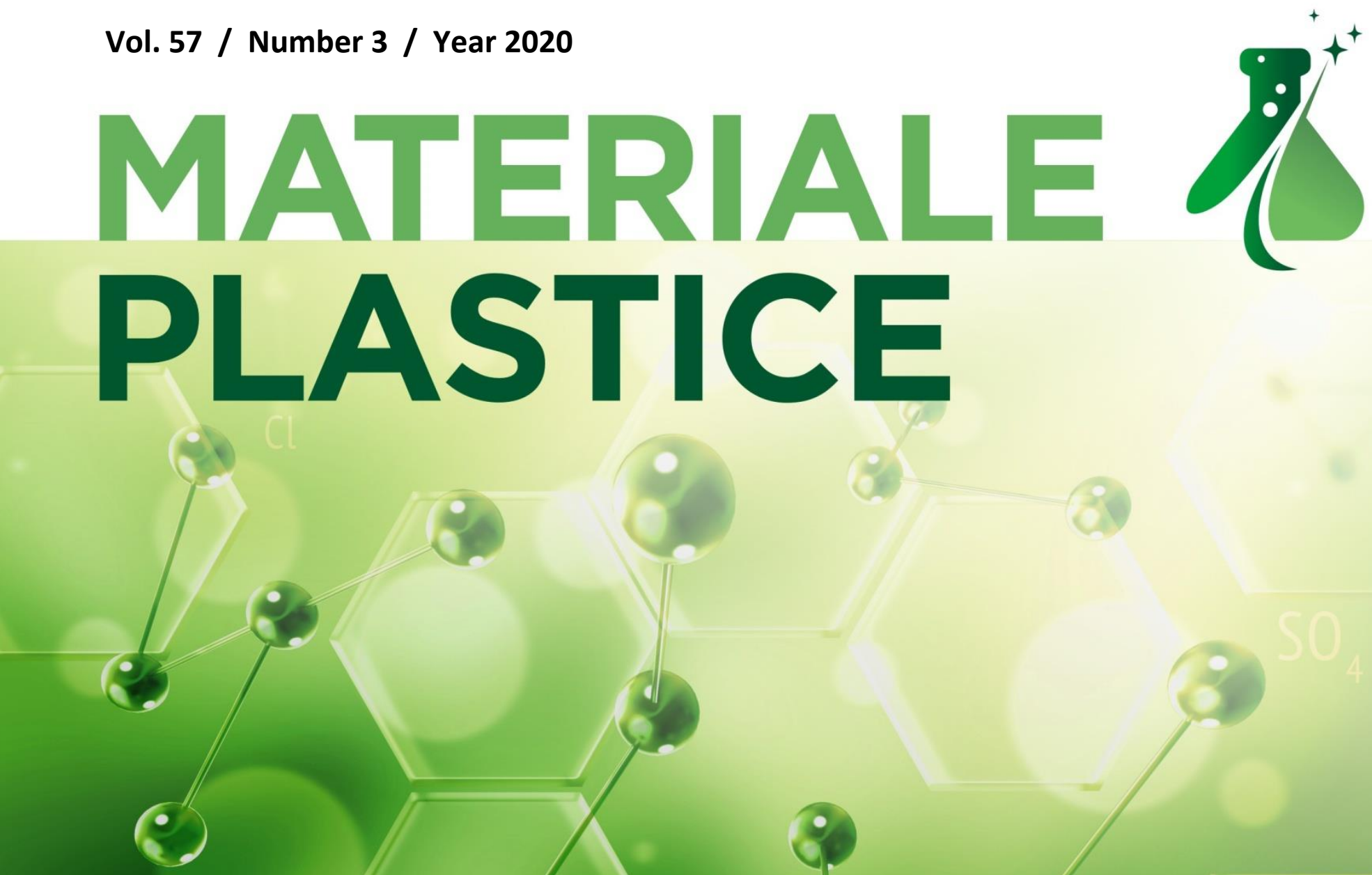

Plastic Maberials

- Flastomers

Synthetic Yarns and Flbers

Composite Materials

Synthesis, characterlzation, Engineering

Applications in Medicine Dental Medicine and Pharmacy

\section{SEPTEMBER 2020}

Print ISSN: 0025 - 5289/ Online ISSN: 2668 - 8220

https://revmaterialeplastice.ro

https://doi.org/10.37358/Mat.Plast.1964

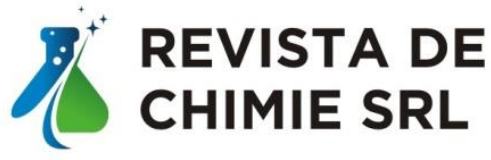


MATERIALE PLASTICE, founded in 1964

edited by the society REVISTA DE CHIMIE S.R.L.

BUCURESTI ROMANIA

\section{EDITORIAL BOARD}

Editor-in-Chief

Horia IOVU

University Politehnica of Bucharest, ROMANIA

\section{Deputy Editor-in-chief \\ Anton HADAR}

University Politehnica of Bucharest, ROMANIA

\section{Deputy Editor-in-chief}

Kamel EARAR

Dunarea de Jos University of Galati, ROMANIA

MEMBERS

Robert ADAMS

Bristol University, UK

Iulian BIRSAN

University Dunarea de Jos of Galati, ROMANIA

Ilare BORDEASU

Politehnica University of Timisoara, ROMANIA

\section{Igor CATIC}

University of Zagreb, CROATIA

Alberto D'AMORE

University of Naples - SUN, Aversa, ITALY

\section{Catalin FETECAU}

University Dunarea de Jos of Galati, ROMANIA

\section{Baki HAZER}

Kapadokya University, TURKEY

Zonguldak Bülent Ecevit University, TURKEY

Cosmin JINESCU

University Politehnica of Bucharest, ROMANIA

Valeriu V. JINESCU

University Politehnica of Bucharest, ROMANIA

\section{Piotr KURCOK}

Centre of Polymer and Carbon Materials Polish Academy of Sciences, POLAND

\section{Gad MAROM}

The Hebrew University of Jerusalem, ISRAEL 
Walter MICHAELI

Institut fur Kunststoffverarbeitung Aachen, GERMANY

George PAPANICOLAOU

University of Patras, GREECE

Carmen RACLES

Petru Poni Institute of Macromolecular Chemistry, Iasi, ROMANIA

Loredana SANTO

University Tor Vergata, Roma, ITALY

Andrei SARBU

INCDCP-ICECHIM Bucuresti, ROMANIA

Ion SERES

S.C. PLASTOR S.A., Oradea, ROMANIA

Bogdan C. SIMIONESCU

Macromolecular Chemistry Institute P. Poni, Iasi, ROMANIA

Norbert STRIBECK

University of Hamburg, GERMANY

Mircea Sava TEODORESCU

University Politehnica of Bucharest, ROMANIA

Nicoleta TEODORESCU

University Politehnica of Bucharest, ROMANIA

Dumitru Mircea VULUGA

Organic Chemistry Center C.D. Nenitescu, Bucharest, ROMANIA

General Manager

Ion ANDRONACHE

Revista de Chimie SRL, Bucharest, ROMANIA

Publisher Chief

Gabriel-Lucian RADU

University Politehnica of Bucharest, ROMANIA

Manager

Alice CRISTEA

Revista de Chimie SRL

Computer Editing

Monica BALUTA

Revista de Chimie SRL

Impact factor 2019: 1.517 


\section{CONTENTS}

\section{ALICIA MENEZES, SHANTHI LYSETTY, ANUP NAHA}

Formulation of Polymeric Nanoparticles of Lercanidipine by Two Bottom Down Techniques Optimized

by Design of Experiment.

ANDREEA IRINA BARZIC, RALUCA MARINICA ALBU, IULIANA STOICA,

BOGDAN OPRISAN

Surface Wettability and Morphology Implications on Interfacial Interactions of Chitosan

with Certain Biological Media.

HAO HUANG, YAN-HUA ZHANG, LI-SHA ZHAO, GUANG-MING LUO, YAN-HUA CAI

Insight into the Role of a Isophthalic Dihydrazide Derivative Containing Piperonylic Acid in

Poly(L-lactide) Nucleation: Thermal Performances and Mechanical Properties.

ALEKSANDRA KALWIK, PRZEMYSLAW POSTAWA, MARCIN NABIALEK

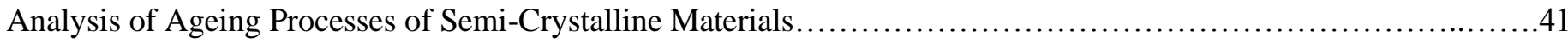

MOHAMED FARSANE, ABDELLAH ANOUAR, SOUAD CHAH, MILOUD BOUZZIRI

Characterization of Ceramic Waste Filled Unsaturated Polyester Resin ...........................................55

GEORGEL MIHU, VASILE BRIA, ADRIAN CIRCIUMARU,

IULIAN GABRIEL BIRSAN, MARINA BUNEA

Specific Heat and Thermal Expansion Coefficient of Hybrid Epoxy Composites

CLAUDIA NICOLETA FEURDEAN, ANIDA-MARIA BABTAN, ANCA IONEL,

ARANKA ILEA, DAN BUHATEL, ARIN SAVA, MARIOARA MOLDOVAN,

CODRUTA SAROSI, STEFAN VESA, RADU SEPTIMIU CAMPIAN

Hybrid Dental Composites Biomechanical Properties.

ALEXANDRINA MUNTEAN, SORINA SAVA, CODRUTA SAROSI, MARIOARA MOLDOVAN,

MARIANA PACURAR, ADA GABRIELA DELEAN

Assessment of Water Sorption, Solubility and Interface Properties for Two Different Pit

and Fissure Sealants.

PETRU BORDEI, CONSTANTIN ANDREI RUSALI, CONSTANTIN IONESCU,

\section{VALERIU ARDELEANU}

Morphometric Characteristics of Renal Vessels and Ureter Concerned on Mulages Obtained

by Plastic Injections.

LUMINITA OANCEA, CORINA MARILENA CRISTACHE, ALEXANDRU EUGEN PETRE,

EUGENIA PANAITESCU, MIHAI BURLIBASA, LUCIAN TOMA CIOCAN

Dimensional Accuracy of Digitalized Stone Models Obtained from Alginic Acid Polymer and

Poly-dimethyl-siloxane Impressions. ...

LIVIU GAVRILA-ARDELEAN, MIHAELA GAVRILA-ARDELEAN

The Flexural Strength of Traditional and Modern Acrylic Prosthetic Bases. ....

DORU MIHAI, NARCISA ELENA POGURSCHI, NICOLETA DANIELA SARBU,

CONSTANTA VALENTINA TUDOR, ADINA HORABLAGA, ROXANA SALCIANU,

LUCIAN CRISTIAN MIRCEA

Polymeric Geomembranes Used in Municipal Waste Facilitie.

TEODOR SANDU, MARIA LUIZA JECU, IULIANA RAUT, MARIANA CALIN,

ELVIRA ALEXANDRESCU, TANTA VERONA IORDACHE,

MARINELA VICTORIA DUMITRU, ANDREI SARBU

Hybrid Beads Bearing Immobilized Bacteria as Advanced Means for the Removal of Acid Blue 93 Dye.

ESRA KUL, FARUK YEŞİLDAL, EMRE MANDEV, CAFER ÇELIKK

Optimization of Variables Influencing the Thermal Conductivity and Fracture Strength of

Reinforced PMMA by Using the Taguchi Method......

MOHAMMAD SAKIB HASAN, TONI IVANOV, MILOS VORKAPIC, ALEKSANDAR SIMONOVIC,

DAVID DAOU, ALEKSANDAR KOVACEVIC, ALEKSA MILOVANOVIC

Impact of Aging Effect and Heat Treatment on the Tensile

Properties of PLA (Poly Lactic Acid) Printed Parts....

NICUSOR BAROIU, GEORGIANA-ALEXANDRA COSTIN, VIRGIL GABRIEL TEODOR,

DUMITRU NEDELCU, VALENTIN TABACARU

Prediction of Surface Roughness in Drilling of Polymers Using

a Geometrical Model and Artificial Neural Networks 
IRINA GRADINARU, LEONARD IGNAT, LEVENTE-CRISTIAN GIURGIU,

CRISTINA GENA DASCALU, LOREDANA LILIANA HURJUI, MAURUSA-ELENA IGNAT,

FLORICA DOROFTEI, ZINOVIA SURLARI, SILVIA FOTEA, GABRIELA GURAU,

ADRIAN BEZNEA, MAGDA-ECATERINA ANTOHE

Study on the Surface Condition of Composite Biomaterials Related to Saliva $p \mathrm{H}$

ROXANA IANCU, STEFAN ANDREI IRIMICIUC, MARICEL AGOP, MIHAIL FRASILA,

MARIA-ALEXANDRA PAUN, VLADIMIR-ALEXANDRU PAUN, VIOREL-PUIU PAUN,

SORIN STRATULAT

5-Fluorouracil Release from Chitosan-Based Matrix. Experimental and Theoretical Aspects

LUCIAN STEFANITA GRIGORE, AMADO-GEORGE STEFAN, OCTAVIAN ORBAN

Using PET-G to Design an Underwater Rover Through 3D Printing Ttechnology....

IONUT LAURENTIU SANDU, FLORIN SUSAC, FELICIA STAN, CATALIN FETECAU

Prediction of Polymer Flow Length by Coupling Finite Element Simulation with Artificial Neural Network

GHEORGHE BATRINESCU, ROXANA-ELENA SCUTARIU, NICOLAE-IONUT CRISTEA,

IOANA-ALEXANDRA IONESCU, GHEORGHE NECHIFOR

The Clogging Effect in the Process of Protein Separation by Ultrafiltration

LAURA-MADALINA CURSARU, ANA-MARIA MOCIOIU, IOAN ALBERT TUDOR, ROXANA MIOARA PITICESCU

Hydrothermal Synthesis of Carbon Nanotubes-Polyaniline (CNT-PANI) Composites and Preliminary

Electrochemical Characterization of CNT-PANI Coatings.

BAIFEN LIU, MOHAMMAD MIRJALILI, PEIMAN VALIPOUR, SAJAD PORZAL, SHIRIN NOURBAKHSH

Morphology and Mechanical Properties of Polyethylene Terephthalate/Ethylene Propylene Diene Monomer (PET/EPDM) in the Presence of Nanoclay.

WAJEEHA FALAH, FU-JIA CHEN, BIBI SAIMA ZEB, MALIK TAHIR HAYAT, QAISAR MAHMOOD, ABDOLGHAFFAR EBAD, MOHSEN TOUGHANI, EN-ZHONG LI

Polyethylene Terephthalate Degradation by Microalga Chlorella vulgaris Along with Pretreatment

VICTOR VLAD COSTAN, NORINA CONSUELA FORNA, OANA ELENA CIURCANU, DANIELA SULEA, ANDRADA DOSCAS, ZENOVIA SURLARI, DAN BOSINCEANU, ADRIAN BEZNEA, GABRIEL VALERIU POPA, SILVIA FOTEA, MIHAI LIVIU CIOFU, SMARANDA BUDURU

Improvements to Biomaterials Structure Used in Acrylic Prostheses. 